\title{
TWO NOTES ON RECURSIVE FUNCTIONS AND REGRESSIVE ISOLS $\left({ }^{(}\right)$
}

\author{
BY \\ JOSEPH BARBACK
}

Introduction. The theory of regressive isols was introduced by J. C. E. Dekker in [7]. The results that we wish to present in this paper belong to this theory and is a continuation of some of our studies in [1], [3] and [4]. We will assume that the reader is familiar with the terminology and some of the main results of the papers listed as references. We let $E$ denote the collection of all nonnegative integers (numbers), $\Lambda$ the collection of all isols, $\Lambda^{*}$ the collection of all isolic integers, and $\Lambda_{R}$ the collection of all regressive isols. If $f$ is a function from a subset of $E$ into $E$ then $\delta f$ will denote its domain and $\rho f$ its range. Let $u_{n}$ and $v_{n}$ be two one-to-one functions from $E$ into $E$. Then $u_{n} \leqq * v_{n}$, if there is a partial recursive function $f$ such that

$$
\rho u \subseteq \delta f \text { and }(\forall n)\left[f\left(u_{n}\right)=v_{n}\right]
$$

In addition, $u_{n}$ and $v_{n}$ are said to be recursively equivalent (denoted $u_{n} \simeq v_{n}$ ), if there is a one-to-one partial recursive function $f$ such that (1) holds. It is easy to see that

$$
u_{n} \simeq v_{n} \Rightarrow \rho u_{n} \simeq \rho v_{n}
$$

Also, it can be shown [8], that

$$
u_{n} \simeq v_{n} \Leftrightarrow\left(u_{n} \leqq * v_{n} \text { and } v_{n} \leqq * u_{n}\right) .
$$

Let $\alpha$ and $\beta$ be two infinite subsets of $E$. Then $\alpha \leqq^{*} \beta$, if there is a partial recursive function $f$ such that $\alpha \subseteq \delta f, f(\alpha)=\beta$ and $f$ is one-to-one on $\alpha$. If $\alpha$ and $\beta$ are each isolated sets then the following is true [8, Proposition P9.(b)],

$$
(\alpha \leqq * \beta \text { and } \beta \leqq * \alpha) \Leftrightarrow \alpha \simeq \beta
$$

Let $\alpha$ and $\beta$ be infinite and isolated regressive sets, and let $a_{n}$ and $b_{n}$ be any regressive functions that range over $\alpha$ and $\beta$ respectively. Then

$$
\alpha \leqq * \beta \Leftrightarrow a_{n} \leqq * b_{n},
$$

Received by the editors April 23, 1968 and, in revised form, October 2, 1968.

(1) The author gratefully acknowledges conversations about some of the topics in the paper with Professors Dekker, Ellentuck, Hassett, Myhill, Nerode and Sansone. The author is an NIH Postdoctoral Fellow in the Department of Statistics (Division of Biostatistics), Stanford University, Stanford, California. Special thanks are expressed for comments of the referee. 
and

$$
\alpha \simeq \beta \Leftrightarrow a_{n} \simeq b_{n}
$$

Property (4) is [8, Proposition P11], and (5) follows directly from (2), (3) and (4). Let $A$ and $B$ be two isols. Then $A \leqq{ }^{*} B$, if either $A$ is a finite isol and $A \leqq B$, or both $A$ and $B$ are infinite isols and there are sets $\alpha$ and $\beta$ belonging to $A$ and $B$ respectively, such that $\alpha \leqq * \beta$. It is readily seen that if both $A$ and $B$ are infinite isols, then $A \leqq * B$ if and only if $\alpha \leqq^{*} \beta$ for any sets $\alpha$ and $\beta$ belonging to $A$ and $B$ respectively. Combining this property with (4), it follows that in the special case both $A$ and $B$ are infinite regressive isols, then $A \leqq * B$ if and only if $a_{n} \leqq{ }^{*} b_{n}$ for all regressive functions $a_{n}$ and $b_{n}$ that range over sets belonging to $A$ and $B$ respectively.

A. Nerode associated with each recursive function $f: E^{n} \rightarrow E$, a function $D_{f}: \Lambda^{n} \rightarrow \Lambda^{*}$, called the canonical extension of $f$ to $\Lambda^{n}$. A discussion of this extension procedure is given in [9, §2] and [15, §4]. A function $f: E \rightarrow E$ is said to be increasing, if for any numbers $x$ and $y, x<y$ implies $f(x) \leqq f(y)$. In the special case that a recursive function $f: E \rightarrow E$ is combinatorial, then $D_{f}: \Lambda \rightarrow \Lambda,[15$, Theorem 6]; and if $f$ is increasing, then $D_{f}: \Lambda_{R} \rightarrow \Lambda_{R}$, [1, Theorem 4]. In [8], J. C. E. Dekker associated with each pair of regressive isols $(A, B)$, a particular regressive isol $\min (A, B)$ called the minimum of $A$ and $B$. The minimum function has the following properties,

$$
\begin{aligned}
& \min (A, B)=\min (B, A), \\
& \min (A, B)=A \Leftrightarrow A \leqq * B .
\end{aligned}
$$

Because there exist regressive isols that are not comparable relative to the relation $\varliminf^{*}$, it can happen that $\min (A, B)$ assumes neither the value $A$ nor the value $B$. Let $D_{\min }(A, B)$ denote the canonical extension to $\Lambda^{2}$ of the ordinary $\min (a, b)$ function from $E^{2}$ into $E$. Then, by [4, p. 15],

$$
\min (A, B)=D_{\min }(A, B), \quad \text { for } A, B \in \Lambda_{R} \text {. }
$$

Properties of regressive isols that are related to the function $\min (A, B)$ and the relation $\varliminf^{*}$ were first studied in [8], and later in [3]. In the first note of this paper we wish to present some additional properties of this kind. We also consider the ordinary recursive function maximum $(a, b)$ for pairs of numbers, and its canonical extension to $\Lambda^{2}$. Some of the main results of the first note are as follows: Let $A$ and $B$ be two regressive isols. Let $f: E \rightarrow E$ be an increasing recursive function, and let $D_{f}$ be its canonical extension to $\Lambda$. Let $\max (A, B)$ denote the canonical extension to $\Lambda^{2}$ of the recursive function maximum $(a, b)$. Then

$$
\begin{aligned}
\min (A, B) & \leqq * A+B, \\
D_{f}(\min (A, B)) & =\min \left(D_{f}(A), D_{f}(B)\right), \\
A \leqq & \Rightarrow D_{f}(A) \leqq D_{f}(B) .
\end{aligned}
$$


It need not be true that max $(A, B)$ will be a regressive isol. However, in the special case that $A+B$ is a regressive isol then $\max (A, B)$ will also be a regressive isol. In this special case the following properties are also shown to be true,

$$
\begin{gathered}
\min (A, B) \leqq A+B, \\
D_{f}(\max (A, B))=\max \left(D_{f}(A), D_{f}(B)\right), \\
D_{f}(A)+D_{f}(B)=D_{f}(\min (A, B))+D_{f}(\max (A, B)), \\
D_{f}(A) \cdot D_{f}(B)=D_{f}(\min (A, B)) \cdot D_{f}(\max (A, B)) .
\end{gathered}
$$

The last two identities turn out to be of interest for the following reason; it is possible to choose regressive isols $A$ and $B$ such that $A+B$ is a regressive isol and such that the four (regressive) isols $D_{f}(A), D_{f}(B), D_{f}(\min (A, B))$, and $D_{f}(\max (A, B))$ are pairwise distinct.

In part II of the paper we study some properties dealing with regressive isols that are prime. Prime isols were first introduced and studied by J. C. E. Dekker and J. Myhill in [10]. Let $w(x)$ denote the strictly increasing recursive function that ranges over the collection of all prime numbers, and let $D_{w}$ denote its canonical extension to $\Lambda$. Because $w$ is an increasing recursive function, $D_{w}$ will map $\Lambda_{R}$ into $\Lambda_{R}$. In the second note we show that $D_{w}$ maps regressive isols onto regressive isols that are prime, and also that there are prime regressive isols that do not belong to $D_{w}\left(\Lambda_{R}\right)$. Our main result gives the existence of four distinct prime regressive isols $U_{1}, U_{2}, V_{1}$ and $V_{2}$ such that

$$
U_{1}, U_{2}, V_{1}, V_{2} \in D_{w}\left(\Lambda_{R}\right), \quad U_{1} \cdot U_{2} \in \Lambda_{R}, \quad \text { and } \quad U_{1} \cdot U_{2}=V_{1} \cdot V_{2} .
$$

It follows therefore, that for regressive isols a unique factorization into primes is not possible.

We wish to acknowledge that throughout the paper an especially important and useful tool is the application of some of the well-known metatheorems, concerning recursive functions and their canonical extensions and isols, of A. Nerode [17].

\section{ON Properties of Regressive Isols}

1. A property of the relation $\leqq *$. In [16], A. Nerode introduced an extension procedure for recursive relations on numbers to relations on isols. This extension procedure is also discussed by E. Ellentuck in [11]. In this section, we wish to prove that between two regressive isols, the extension to the isols of the ordinary relation $\leqq$ between two numbers, is equivalent to the relation $\leqq *$. We first describe how the extension to the isols of a recursive binary relation can be characterized. Let $\Delta \subseteq E^{2}$ be a recursive binary relation, and let $f, g: E^{2} \rightarrow E$ be any recursive functions such that

$$
\Delta=\{(x, y) \mid x, y \in E \text { and } f(x, y)=g(x, y)\} .
$$


Then the extension of the relation $\Delta$ to $\Lambda$ can be characterized as the set

$$
\Delta_{\Lambda}=\left\{(X, Y) \mid X, Y \in \Lambda \text { and } D_{f}(X, Y)=D_{g}(X, Y)\right\} \text {. }
$$

Let $\Delta_{R}$ denote the binary relation on $\Lambda_{R}$ that is defined by

$$
\Delta_{R}=\Delta_{\Lambda} \cap \Lambda_{R}^{2}
$$

THEOREM 1.1. Let $\leqq_{\Lambda}$ denote the extension to $\Lambda$ of the ordinary relation $\leqq$ between two numbers. Let $\leqq_{R}$ denote the relation on $\Lambda_{R}$ obtained from $\leqq_{\Lambda}$ by (1.2). Then for all regressive isols $A$ and $B$,

$$
A \leqq_{R} B \Leftrightarrow A \leqq * B .
$$

Proof. Let $\Delta$ denote the relation $\leqq$. Then

$$
\begin{aligned}
\Delta & =\{(x, y) \mid x, y \in E \text { and } x \leqq y\} \\
& =\{(x, y) \mid x, y \in E \text { and } \min (x, y)=x\} .
\end{aligned}
$$

Therefore

$$
\Delta_{\Lambda}=\left\{(X, Y) \mid X, Y \in \Lambda \text { and } D_{\min }(X, Y)=X\right\} .
$$

Combining (7), (8), (1.2) and (1.3) we see that

$$
\begin{aligned}
\Delta_{R} & =\left\{(A, B) \mid A, B \in \Lambda_{R} \text { and } D_{\min }(A, B)=A\right\} \\
& =\left\{(A, B) \mid A, B \in \Lambda_{R} \text { and } \min (A, B)=A\right\} \\
& =\left\{(A, B) \mid A, B \in \Lambda_{R} \text { and } A \leqq^{*} B\right\} .
\end{aligned}
$$

The desired result follows directly from the last equality, and this completes the proof.

2. Some fundamental properties of $\min (A, B)$. In [8], Dekker proved the following three properties;

$$
\begin{gathered}
A \leqq B, \quad B \in \Lambda_{R} \Rightarrow A \in \Lambda_{R}, \\
A+B \in \Lambda_{R} \Leftrightarrow A \cdot B \in \Lambda_{R}, \quad \text { when } A, B \geqq 1, \\
\left(\exists A, B \in \Lambda_{R}\right)\left[A+B \notin \Lambda_{R}\right]^{\prime \prime}
\end{gathered}
$$

In contrast to the isols, the regressive isols are neither closed under addition nor multiplication. One of the main goals of [3] was to try to obtain useful necessary conditions and sufficient conditions on two regressive isols for their sum to be a regressive isol. The next two theorems that we wish to prove are in a manner related to this goal.

TheOREM 2.1. Let $A$ and $B$ be any regressive isols. Then
(a) $\min (A, B) \leqq * A+B$,
(b) $A+B \in \Lambda_{R} \Rightarrow \min (A, B) \leqq A+B$. 
THEOREM 2.2. There are (infinite) regressive isols $A$ and $B$, for which the relation $\min (A, B) \leqq A+B$ is not true.

Remark. Part (a) of Theorem 2.1 is a result due to W. Richter. Part (b) of Theorem 2.1 was stated without proof in [3], and its proof is related to the proof of [3, Theorem 1.2].

Proof of Theorem 2.1. If either of the regressive isols $A$ or $B$ is finite, then the value of $\min (A, B)$ will equal $A$ or equal $B$. In this event both parts of the theorem are readily seen to be true.

Let us assume now that both $A$ and $B$ are infinite regressive isols. Let $a_{n}$ and $b_{n}$ be regressive functions that range over the sets $\alpha$ and $\beta$ respectively, and with $\alpha \in A, \beta \in B$ and $\alpha \mid \beta$. Then $\alpha+\beta \in A+B$.

$\operatorname{Re}(\mathrm{a})$. Let $d_{n}=j\left(a_{n}, b_{n}\right)$ for $n \in E$. Then $d_{n}$ is a regressive function and will range over a set in $\min (A, B)$. Let $\partial$ denote the range of $d_{n}$. Consider the mapping from $\partial$ onto $\alpha+\beta$ given by

$$
\begin{aligned}
& d_{n}=j\left(a_{n}, b_{n}\right) \rightarrow a_{k}, \quad \text { if } n=2 k, \\
& \rightarrow b_{k}, \quad \text { if } n=2 k+1 \text {. }
\end{aligned}
$$

Taking into account that $j$ is a one-to-one recursive function and that each of the functions $a_{n}, b_{n}$ and $d_{n}$ is regressive, it is readily seen that the mapping denoted by $\left(^{*}\right)$ has a partial recursive extension. This property implies that $\partial \leqq^{*} \alpha+\beta$, and therefore also that $\min (A, B) \leqq * A+B$.

$\operatorname{Re}$ (b). Assume $A+B \in \Lambda_{R}$. Then $\alpha+\beta$ will be an (infinite) regressive set. Let $c_{n}$ be a regressive function that ranges over $\alpha+\beta$, and let $p(x)$ be a (partial recursive) regressing function for $c_{n}$. Let $p^{*}$ be a partial recursive function associated with $p$ that has the property, $p^{*}\left(c_{n}\right)=n$ for $n \in E$. We note that for each number $n \in E$, exactly one of the two relations $p^{*}\left(a_{n}\right)<p^{*}\left(b_{n}\right)$ or $p^{*}\left(b_{n}\right)<p^{*}\left(a_{n}\right)$ will be true. Let

$$
\delta=\left\{x \mid\left(x=a_{n} \text { and } p^{*}\left(b_{n}\right)<p^{*}\left(a_{n}\right)\right) \bigvee\left(x=b_{n} \text { and } p^{*}\left(a_{n}\right)<p^{*}\left(b_{n}\right)\right)\right\}
$$

Then $\delta \subseteq \alpha+\beta$ and for each number $n \in E$, exactly one of the two numbers $a_{n}$ or $b_{n}$ will belong to $\delta$.

We now verify,

(b.1) $\delta \mid(\alpha+\beta)-\delta$,

(b.2) $\delta \in \min (A, B)$.

For (b.1). Let $x \in \alpha+\beta$. We wish to determine whether or not $x \in \delta$. Since $\alpha$ and $\beta$ are separated sets we can find out whether $x \in \alpha$ or $x \in \beta$. First suppose that $x \in \alpha$. Because $a_{n}$ and $c_{n}$ are regressive functions, we can compute the numbers $u$ and $v$ such that $x=a_{u}=c_{v}$. In view of the definition of $\delta$, we see that

$$
a_{u} \in \delta \Leftrightarrow b_{u} \in\left(c_{0}, \ldots, c_{v-1}\right)=\left\{p^{r}(x) \mid 1 \leqq r \leqq v\right\} .
$$

The members of the set on the right side of (2.4) can be effectively obtained from $x$, since $p$ is a partial recursive function. In addition, using again the separability of the sets $\alpha$ and $\beta$, and the regressive property of the function $b_{n}$, we can determine 
whether or not the number $b_{u}$ belongs to the set $\left(c_{0}, \ldots, c_{v-1}\right)$. In view of (2.4), it follows that we can therefore also determine whether or not the number $x$ belongs to $\delta$. We recall that we had assumed that $x \in \alpha$. It can be readily verified in a similar fashion that one can determine whether or not $x \in \delta$, in the event $x \in \beta$. We can conclude from these remarks that the two sets $\delta$ and $(\alpha+\beta)-\delta$ are separated.

For (b.2). Let the functions $u_{n}$ and $v_{n}$ be defined by

and

$$
\begin{aligned}
u_{n}=a_{n}, & \text { if } a_{n} \in \delta, \\
=b_{n}, & \text { if } b_{n} \in \delta ;
\end{aligned}
$$

$$
\begin{aligned}
v_{n}=a_{n}, & \text { if } a_{n} \notin \delta, \\
=b_{n}, & \text { if } b_{n} \notin \delta .
\end{aligned}
$$

Then each of the functions $u_{n}$ and $v_{n}$ is everywhere defined and one-to-one; also $u_{n}$ ranges over the set $\delta$ and $v_{n}$ ranges over the set $(\alpha+\beta)-\delta$. Let $f$ be the partial function with domain $\delta$ and defined by, $f\left(u_{n}\right)=v_{n}$. In the course of proving [3, Theorem 1.2] it was verified that the mapping $f$ will have a partial recursive extension. It follows from this property that

$$
u_{n} \leqq v_{n}
$$

Because $d_{n}=j\left(a_{n}, b_{n}\right)$ is a (regressive) function that ranges over a set in $\min (A, B)$, and $u_{n}$ is a function that ranges over the set $\delta$, to establish (b.2) we see that it is sufficient to verify

$$
u_{n} \simeq j\left(a_{n}, b_{n}\right) \text {. }
$$

This will be our approach here. We first note that (2.6) is equivalent to the two relations

$$
u_{n} \leqq j\left(a_{n}, b_{n}\right)
$$

and

$$
j\left(a_{n}, b_{n}\right) \leqq * u_{n}
$$

The first of these follows directly from (2.5) and the definition of the functions $u_{n}$ and $v_{n}$. To verify (2.8), we first recall that for each number $n$, either $u_{n}=a_{n}$ or $u_{n}=b_{n}$. It readily follows from our remarks in the proof of (b.1), that given both of the numbers $a_{n}$ and $b_{n}$, we can effectively determine which one is $u_{n}$. Since both $a_{n}$ and $b_{n}$ can be found from the value of $j\left(a_{n}, b_{n}\right)$, it follows therefore that the mapping $j\left(a_{n}, b_{n}\right) \rightarrow u_{n}$ will have a partial recursive extension. This property is equivalent to the relation of (2.8). This verifies both (2.7) and (2.8); and as we noted earlier, together these establish both (2.6) and (b.2). This completes the proof.

REMARK. Regarding the proof of Theorem 2.1(b), we wish to note here some properties of the two functions $u_{n}$ and $v_{n}$, that are of interest in the next section. These are 
(a) $u_{n}$ and $v_{n}$ range over separated sets,

(b) $u_{n}$ ranges over a set in $\min (A, B)$, and

(c) $u_{n}$ and $v_{n}$ are each regressive functions.

Because the functions $u_{n}$ and $v_{n}$ range over the sets $\delta$ and $(\alpha+\beta)-\delta$ respectively, we see that (a) and (b) follow directly from (b.1) and (b.2) respectively. To verify (c), we first note that by combining (2.6) and the fact the function $d_{n}=j\left(a_{n}, b_{n}\right)$ is regressive, it follows that $u_{n}$ will be a regressive function. We now want to show that the function $v_{n}$ is also regressive, i.e., that the mapping $v_{n+1} \rightarrow v_{n}$ will have a partial recursive extension. For this purpose, let the value of $v_{n+1}$ be given; we wish to find the value of $v_{n}$. We know that for $m \in E$,

$$
\begin{aligned}
& v_{m}=a_{m}, \quad \text { if } a_{m} \notin \delta, \\
& =b_{m}, \quad \text { if } b_{m} \notin \delta \text {. }
\end{aligned}
$$

Since $\alpha$ and $\beta$ are separated sets we can effectively determine whether $v_{n+1}=a_{n+1}$ or $v_{n+1}=b_{n+1}$. Assume that $v_{n+1}=a_{n+1}$. Then, using the regressive property of the function $a_{x}$, we can find the value of $a_{n}$. In view of (b.1), we can then determine whether or not $a_{n} \in \delta$. If $a_{n} \notin \delta$, then $v_{n}=a_{n}$ and we are done. In view of (2.5), we see that if $a_{n} \in \delta$ we can then also find the value of $v_{n}$. We had assumed that $v_{n+1}=a_{n+1}$, and then showed that the value of $v_{n}$ could be found. It is easy to see that we could also have found the value of $v_{n}$ if it turned out that $v_{n+1}=b_{n+1}$. We can conclude from these remarks that the mapping $v_{n+1} \rightarrow v_{n}$, will have a partial recursive extension, and therefore also that $v_{n}$ is a regressive function.

In [8], Dekker introduced the concept of the degree of unsolvability of a regressive isol. This is a useful notion and is employed in the proof of Theorem 2.2. We now recall, from [8], how it is defined. Every regressive isol contains at least one retraceable set. By [8, Corollary 2 of Proposition P14], any two retraceable sets that belong to the same isol [i.e., that are recursively equivalent] will have the same (Turing) degree of unsolvability. Let $A$ be a regressive isol. Then its degree of unsolvability, denoted by $\Delta_{A}$, is defined to be the (Turing) degree of unsolvability of any retraceable set belonging to $A$. In the special case $A$ and $B$ are infinite regressive isols, then the degree has the following properties [8],

(i) $A+B \in \Lambda_{R} \Rightarrow \Delta_{A}=\Delta_{B}$,

(ii) $A \leqq B \Rightarrow \Delta_{A}=\Delta_{B}$,

(iii) $\Delta_{A} \leqq \Delta_{\min (A, B)}$ and $\Delta_{B} \leqq \Delta_{\min (A, B)}$.

In addition, it was also proved in [8], that there exist infinite regressive isols that have degrees incomparable with respect to the relation $\leqq$.

LEMMA 2.1. Let $A$ and $B$ be infinite regressive isols. Then

$$
\min (A, B) \leqq A+B \Rightarrow \Delta_{A} \leqq \Delta_{B} \text { or } \Delta_{B} \leqq \Delta_{A} .
$$

Proof. We note that $\min (A, B)$ is an infinite (regressive) isol, since each of the isols $A$ and $B$ is infinite. Assume that

$$
\min (A, B) \leqq A+B .
$$


Combining (2.1) and the refinement property for recursive equivalence types, it follows from (2.9) that there will exist regressive isols $M$ and $N$ such that

$$
M+N=\min (A, B), \quad M \leqq A \text { and } N \leqq B .
$$

Because $\min (A, B)$ is infinite, at least one of the two regressive isols $M$ and $N$ is also infinite. Let us assume that $M$ is infinite. Then $M \leqq A$ implies, by (ii), that $\Delta_{M}=\Delta_{A}$, and $M \leqq \min (A, B)$ implies that $\Delta_{M}=\Delta_{\min (A, B)}$. Therefore $\Delta_{A}=\Delta_{\min (A, B)}$. Combining this identity with (iii), gives $\Delta_{B} \leqq \Delta_{A}$. In a similar manner one can show that if $N$ is infinite then $\Delta_{A} \leqq \Delta_{B}$. This gives the desired conclusion that $\Delta_{A}$ and $\Delta_{B}$ are comparable degrees with respect to the $\leqq$ relation.

Proof of Theorem 2.2. Use Lemma 2.1 and the fact that there exist infinite regressive isols that have incomparable degrees of unsolvability.

REMARK. It is proven in [8] that there are infinite cosimple regressive isols that have incomparable degrees of unsolvability. Also, the minimum of two cosimple regressive isols is again a cosimple regressive isol. Thus, as a consequence of the previous lemma, we see that the following result is also true.

THEOREM. There are (infinite) cosimple regressive isols $A$ and $B$, for which the relation $\min (A, B) \leqq A+B$ is not true.

We state the next result without proof. It is a special case of a theorem of Nerode.

LEMmA A. Let $f: E^{n} \rightarrow E$ and $g_{1}, \ldots, g_{n}: E^{m} \rightarrow E$ be recursive functions. Let $f \circ g$ denote the composition function $f\left(g_{1}(X), \ldots, g_{n}(X)\right)$, for $X \in E^{m}$. Then $f \circ g$ is a recursive function, and

$$
D_{f \circ g}(T)=D_{f}\left(D_{g_{1}}(T), \ldots, D_{g_{n}}(T)\right), \text { for } T \in \Lambda^{m} \text { and } D_{g_{1}}(T), \ldots, D_{g_{n}}(T) \in \Lambda \text {. }
$$

THEOREM 2.3. Let $f: E \rightarrow E$ be an increasing recursive function, and $A$ and $B$ any regressive isols. Then

(a) $\min \left(D_{f}(A), D_{f}(B)\right)=D_{f}(\min (A, B))$,

(b) $A \leqq{ }^{*} B \Rightarrow D_{f}(A) \leqq * D_{f}(B)$.

Proof. Because $f$ is an increasing recursive function, we know that both $D_{f}(A)$ and $D_{f}(B)$ will be regressive isols; the identity of part (a) is therefore meaningful.

$\operatorname{Re}(\mathrm{a})$. Let $\min (a, b)$ denote the familiar minimum function from $E^{2}$ into $E$. Then

$$
\min (f(a), f(b))=f(\min (a, b)), \quad \text { for } a, b \in E .
$$

Application of Lemma A to the identity of (2.10) gives

$$
D_{\min }\left(D_{f}(A), D_{f}(B)\right)=D_{f}\left(D_{\min }(A, B)\right) .
$$

Finally, combining (8) and (2.11) yields (a).

$\operatorname{Re}$ (b). Assume that $A \leqq{ }^{*} B$. Then $\min (A, B)=A$ and therefore also

$$
D_{f}(\min (A, B))=D_{f}(A) \text {. }
$$


Combining part (a) and (2.12) gives

$$
\min \left(D_{f}(A), D_{f}(B)\right)=D_{f}(A) ;
$$

and this implies that $D_{f}(A) \leqq{ }^{*} D_{f}(B)$. This verifies (b) and completes the proof.

REMARK. As an illustration of Theorem 2.3 we mention

and

$$
\min \left(2^{A}, 2^{B}\right)=2^{\min (A, B)}
$$

$$
A \leqq * B \Rightarrow 2^{A} \leqq * 2^{B}, \text { for } A, B \in \Lambda_{R} .
$$

The reader is referred to $[8, \mathrm{p} .365]$ for some additional comments that are related to Theorem 2.3. In the special case that $f$ is a strictly increasing recursive function, then the converse of Theorem 2.3(b) is also true, and this will be our next result. We need the following lemma; it is a special case of a theorem of Nerode and we shall state it without proof.

LEMMA B. Let $f, g, h$ and $k$ be recursive functions from $E^{n}$ into $E$. If

then

$$
\left(\forall t \in E^{n}\right)[f(t)=g(t) \Rightarrow h(t)=k(t)],
$$

$$
\left(\forall T \in \Lambda^{n}\right)\left[D_{f}(T)=D_{g}(T) \Rightarrow D_{h}(T)=D_{k}(T)\right] .
$$

THEOREM 2.4. Let $f: E \rightarrow E$ be a strictly increasing recursive function, and $A$ and $B$ any regressive isols. Then $D_{f}(A) \leqq{ }^{*} D_{f}(B) \Rightarrow A \leqq{ }^{*} B$.

Proof. Because $f$ is a strictly increasing function, it follows that for any numbers $a, b \in E, f(a) \leqq f(b) \Rightarrow a \leqq b$, and therefore also,

$$
\min (f(a), f(b))=f(a) \Rightarrow \min (a, b)=a .
$$

In view of Lemmas $A$ and $B$, it follows from (2.13) that

$$
\min \left(D_{f}(A), D_{f}(B)\right)=D_{f}(A) \Rightarrow \min (A, B)=A .
$$

Because $f$ is an increasing recursive function, we know that $D_{f}(A)$ and $D_{f}(B)$ are regressive isols. Therefore, by (7), we see that (2.14) is equivalent to $D_{f}(A) \leqq{ }^{*} D_{f}(B)$ $\Rightarrow A \leqq * B$, and this is the desired result.

REMARK. Let $f: E \rightarrow E$ be a strictly increasing recursive function and let $A$ and $B$ be any regressive isols. By Theorems 2.3(b) and 2.4 we know that

and

$$
A \leqq * B \Rightarrow D_{f}(A) \leqq * D_{f}(B),
$$

$$
D_{f}(A) \leqq D_{f}(B) \Rightarrow A \leqq * B .
$$

We wish to consider the following two similar assertions,

(I) $A \leqq B \Rightarrow D_{f}(A) \leqq D_{f}(B)$,

(II) $D_{f}(A) \leqq D_{f}(B) \Rightarrow A \leqq B$.

Concerning (I), let the function $\tilde{f}^{x}$ be defined by, $f^{f}(n)=f(n+1)-f(n)$ for $n \in E$. Then $f$ will be a recursive function, and it is called the first difference function of $f$. 
In [20], Sansone proved that if $\tilde{f}$ is an eventually increasing function then statement (I) is true. He also showed that in the event $\tilde{f}$ is not an eventually increasing function that not only will (I) not be a theorem, but that there will even exist an (infinite) regressive isol $T$ such that $D_{f}(T) \leqq D_{f}(T+1)$ is not true. It can be readily shown that in the special case that $f$ is a combinatorial function, then its first difference function $f^{f}$ is also a combinatorial function; and therefore also an increasing function. In view of these remarks, we see that while statement (I) is not true for all strictly increasing recursive functions $f$, it is true in the particular case that $f$ is combinatorial.

Concerning statement (II), (II) will be true in the special case that $f$ is a linear function, i.e., $f(x)=a x+b$, where $a$ and $b$ denote some particular constants. This property follows from a result of Nerode (see [12, p. 1]). On the other hand, Ellentuck proved in [12, Theorem 3] that if $f$ is a combinatorial but nonlinear function, then there are isols $A$ and $B$ for which statement (II) is false. We wish to note that such isols $A$ and $B$ can be chosen to be regressive. [To obtain this last property, simply modify the proof of [12, Theorem 3] by selecting $U$ to be a universal regressive isol. The existence of universal isols that are regressive was recently proved by Ellentuck in some notes not yet published.]

3. $\max (A, B)$. Let the ordinary recursive function, maximum $(a, b)$, be denoted by $\max (a, b)$ for pairs of numbers, and let $\max (A, B)$ denote its canonical extension, $D_{\max }(A, B)$, to $\Lambda^{2}$. It is easy to show that $\max (a, b)$ is not an almost combinatorial function; and therefore $\max (A, B)$ will not map $\Lambda^{2}$ into $\Lambda$. It is also true that $\max (A, B)$ will not map $\Lambda_{R}^{2}$ into $\Lambda_{R}$; M. Hassett proved this property, in [13]. However, in the special case that $A$ and $B$ are regressive isols such that their sum $A+B$ is also regressive, then $\max (A, B)$ as well as $\min (A, B)$ will be regressive isols. To prove this fact, first note that the statement

$$
\min (a, b)+c=a+b \Rightarrow c=\max (a, b)
$$

is valid in the domain $E$. Hence, by Lemma B,

$$
\min (A, B)+C=A+B \Rightarrow C=\max (A, B), \text { for } A, B \in \Lambda_{R} \text {. }
$$

By Theorem 2.1(b),

$$
\min (A, B) \leqq A+B, \quad \text { when } A, B, A+B \in \Lambda_{R}
$$

Combining (3.1) and (3.2), we see that in the special case $A, B$ and $A+B$ are regressive isols, then

$$
\max (A, B)=(A+B)-\min (A, B), \quad \text { and } \max (A, B) \in \Lambda_{R} .
$$

Notation. For the remainder of this section we let $A$ and $B$ denote any regressive isols such that $A+B \in \Lambda_{R}$. By our previous remarks we then have that both $\min (A, B)$ and $\max (A, B)$ will be regressive isols. 
REMARK. In view of (3.1), and properties of the minimum of two regressive isols, it follows that the values of $\min (A, B)$ and $\max (A, B)$ will be closely related. For example, it is readily shown that

(a)

and

$$
\begin{aligned}
\max (A, B) & =\max (B, A), \\
\max (A, B) \in(A, B) & \Leftrightarrow \min (A, B) \in(A, B),
\end{aligned}
$$

In the course of proving Theorem 2.1(b) we assumed that $A$ and $B$ were infinite regressive isols such that $A+B \in \Lambda_{R}$, and then we proved that $\min (A, B) \leqq A+B$. In doing this we defined two (one-to-one) functions $u_{n}$ and $v_{n}$. In the remark following the proof of Theorem 2.1, we observed that both $u_{n}$ and $v_{n}$ are regressive functions, and that $u_{n}$ ranges over a set in $\min (A, B)$. In addition, the ranges of the functions $u_{n}$ and $v_{n}$ are separated sets. In view of (3.1), it follows from this fact that the (regressive) function $v_{n}$ ranges over a set in $\max (A, B)$. If one combines these properties with the definitions of the functions $u_{n}$ and $v_{n}$, then the following properties can be readily established:

$$
\begin{gathered}
A, B \in \Lambda_{R}-E \Rightarrow \min (A, B), \quad \max (A, B) \in \Lambda_{R}-E, \\
A \leqq * \max (A, B) \quad \text { and } B \leqq \\
\min (A, B)=\max (A, B),
\end{gathered}
$$

and

$(\eta)$

$$
\left(\forall T \in \Lambda_{R}\right)[A \leqq * T \text { and } B \leqq * T \Leftrightarrow \max (A, B) \leqq * T] .
$$

We note that properties $(\varepsilon)$ and $(\eta)$ represent analogues for the function max $(A, B)$ of two well-known properties of $\min (A, B),[8, \mathrm{p}$. 363]. The next theorem we state without proof, because each of its parts can be verified in a manner similar to that employed in the proof of Theorem 2.3(a).

THEOREM 3.1. Let $f: E \rightarrow E$ be an increasing recursive function. Then

(a) $\max \left(D_{f}(A), D_{f}(B)\right)=D_{f}(\max (A, B))$,

(b) $\min (A, B) \cdot \max (A, B)=A \cdot B$,

(c) $\min \left(D_{f}(A), D_{f}(B)\right)+\max \left(D_{f}(A), D_{f}(B)\right)=D_{f}(A)+D_{f}(B)$,

(d) $\min \left(D_{f}(A), D_{f}(B)\right) \cdot \max \left(D_{f}(A), D_{f}(B)\right)=D_{f}(A) \cdot D_{f}(B)$,

(e) $D_{f}(\min (A, B)) \cdot D_{f}(\max (A, B))=D_{f}(A) \cdot D_{f}(B)$.

\section{Prime Regressive Isols}

Let $U$ be an isol; we recall, from [10], the following definitions,

$U$ is multiple-free if $2 A \leqq U \Rightarrow A \in E$,

$U$ is prime if $A \mid U \Rightarrow(A=1$ or $A=U)$. 
Each of the results

$$
\text { infinite multiple-free isols exist }
$$

and

$$
\text { infinite multiple-free isols are prime }
$$

is proved in [10].

THEOREM 4.1. There is an infinite regressive isol $T$ that is multiple-free.

Proof. This will be a constructive type of proof and we will use a technique introduced in the proof of [10, Theorem 95].

Let $\left\{p_{i}\right\}$ be an enumeration of all partial recursive functions of one variable that are one-to-one [i.e., that are one-to-one on their respective domains]. For each number $i$, we let $p_{i}^{-1}$ denote the inverse function of $p_{i}$. We note that for each $i$, the function $p_{i}^{-1}$ is also partial recursive and one-to-one. If $p$ is any partial function, $x$ any number, and $\alpha$ any set of numbers, then we will write $p(x) \notin \alpha$ to mean either, $p(x)$ is undefined, or $p(x)$ is defined and $p(x) \notin \alpha$. We shall now define a function $t_{n}$ such that the recursive equivalence type $T=\operatorname{Req} \rho t_{n}$ will satisfy the condition of the theorem.

Set $t_{0}=1$. Let $n \geqq 1$ and assume that the values $t_{0}, \ldots, t_{n-1}$ have already been defined. Let

$$
\pi_{n-1}=\left(t_{0}, \ldots, t_{n-1}\right)
$$

We define $t_{n}$ by setting

$$
t_{n}=j\left(t_{n-1}, u_{n}\right)
$$

where the number $u_{n}$ is given by,

$$
u_{n}=(\mu n)\left[(\forall r \leqq n)\left[p_{r} j\left(t_{n-1}, u\right) \notin \pi_{n-1} \text { and } p_{r}^{-1} j\left(t_{n-1}, u\right) \notin \pi_{n-1}\right]\right] .
$$

In light of the fact that each of the functions $p_{i}$ and $j$ is one-to-one, it is readily seen that there will be a number $u_{n}$ satisfying (4.5). This completes the definition of the function $t_{n}$. In almost the same fashion as in the proof of [1, Theorem 4], one can easily verify that the function $t_{n}$ has the following properties,

$$
\begin{aligned}
& t_{n} \text { is everywhere defined and one-to-one, } \\
& t_{n} \text { is a retraceable function. }
\end{aligned}
$$

Let

$$
\tau=\rho t_{n} \text { and } \quad T=\operatorname{Req} \tau .
$$

Then $\tau$ is an infinite retraceable set and $T$ is an infinite regressive RET.

We now verify that,

(a) $T$ is multiple-free,

(b) $\tau$ is an immune set,

(c) $T$ is an infinite multiple-free regressive isol. 
$\operatorname{Re}(\mathrm{a})$. Let us assume otherwise, and let $A$ denote an RET such that

$A$ is infinite and $2 A \leqq T$.

Then both $A$ and $2 A$ will be (infinite) regressive RET's. In addition, there will exist infinite regressive sets $\alpha, \alpha^{*} \subseteq \tau$ such that,

$$
\alpha \cap \alpha^{*}=\varnothing \quad \text { and } \alpha, \alpha^{*} \in A \text {. }
$$

Let $u_{n}$ and $v_{n}$ be one-to-one functions such that

$$
\begin{gathered}
\rho u_{n} \cap \rho v_{n}=\varnothing, \\
\rho t_{u_{n}}=\alpha \text { and } \rho t_{v_{n}}=\alpha^{*}
\end{gathered}
$$

and

$$
t_{u_{n}} \text { and } t_{v_{n}} \text { are each regressive functions. }
$$

Because $\alpha, \alpha^{*} \in A$, it follows that $\alpha \simeq \alpha^{*}$. Combining this fact with (4.10), (4.11) and [7, Proposition 3], it follows that the two regressive functions $t_{u_{n}}$ and $t_{v_{n}}$ are recursively equivalent; this means that there will exist a one-to-one partial recursive function $p$ such that, for each number $n \in E$,

$$
p\left(t_{u_{n}}\right) \text { is defined, and } p\left(t_{u_{n}}\right)=t_{v_{n}} \text {. }
$$

Such a function $p$ will appear in our enumeration $\left\{p_{i}\right\}$; let $i$ be one of its indices. In view of (4.12), it then follows that, for each number $n \in E$,

$$
p_{i}\left(t_{u_{n}}\right)=t_{v_{n}} \text { and } p_{i}^{-1}\left(t_{v_{n}}\right)=t_{u_{n}}
$$

Let $m$ be the smallest number such that $u_{m}, v_{m}>i$. By (4.9), we note that $u_{m} \neq v_{m}$. We consider two cases:

Case 1. $u_{m}<v_{m}$. In light of the definition of the function $t_{n}$, parts (4.3), (4.4) and (4.5), it follows then that

$$
p_{i}^{-1}\left(t_{v_{m}}\right) \notin\left(t_{0}, \ldots, t_{v_{m-1}}\right),
$$

and therefore also

$$
p_{i}^{-1}\left(t_{v_{m}}\right) \notin\left(t_{u_{0}}, \ldots, t_{u_{m}}\right) .
$$

Hence $p_{i}^{-1}\left(t_{v_{m}}\right) \neq t_{u_{m}}$, which cannot be true in view of (4.13).

Case 2. $u_{m}>v_{m}$. Then, as in the previous case, it follows that

$$
p_{i}\left(t_{u_{m}}\right) \notin\left(t_{0}, \ldots, t_{u_{m-1}}\right),
$$

and

$$
p_{i}\left(t_{u_{m}}\right) \notin\left(t_{v_{0}}, \ldots, t_{v_{m}}\right) .
$$

The last relation implies $p_{i}\left(t_{u_{m}}\right) \neq t_{v_{m}}$, and this also cannot be true in view of (4.13). 
In view of our remarks in each of these cases, we see that there does not exist a one-to-one partial recursive function $p$ that satisfies (4.12). We can therefore conclude that $T$ is a multiple-free RET; and this verifies part (a).

$\operatorname{Re}(\mathrm{b})$. We have already noted that $\tau$ is an infinite retraceable set. It is well known that retraceable sets are recursive or immune. If $\tau$ were a recursive set, then the strictly increasing function that ranges over $\tau$, namely $t_{n}$, would be a recursive function. In this event, it would then be an easy consequence that $t_{2 n} \simeq t_{2 n+1}$, which would imply that $T$ is an even RET. However, this is not possible, since $T$ is infinite and, by part (a), a multiple-free RET. We can conclude therefore that $\tau$ will be an immune set.

$\operatorname{Re}(\mathrm{c})$. Because $T=\operatorname{Req} \tau$, and $\tau$ is an infinite retraceable and immune set, it follows that $T$ is an infinite regressive isol. Combining this fact with part (a), gives that $T$ is an infinite multiple-free regressive isol. This verifies part (c) and completes the proof of the theorem.

COROLlaRY 4.1. There exist infinite regressive isols that are prime.

Proof. Combine Theorem 4.1 and (4.2).

REMARK. Let $T$ be an infinite multiple-free regressive isol. Let $n \in E$. It can be readily verified that each isol of the form $T+n$ and $T-n$, is also regressive and multiple-free. In view of (4.2), we see then that each isol in the enumeration

$$
\ldots, T-2, T-1, T, T+1, T+2, \ldots,
$$

will be infinite, regressive and prime. It is interesting to note here that even though both $T$ and $T+1$ will be infinite prime isols, the number 2 will be a factor of the product $T \cdot(T+1)$ [10, Theorem 73].

NotATION AND REMARK. For the remainder of the paper we let $w(x)$ be the function given by $w(x)=x$ th prime number, i.e., $w(0)=2, w(1)=3, \ldots$ Because $w(x)$ is a (strictly) increasing recursive function, its canonical extension $D_{w}$ will map $\Lambda_{R}$ into $\Lambda_{R}$. However, its extension will not map $\Lambda$ into $\Lambda$. The reason for this is that $w(x)$ will not be an eventually combinatorial function [this property was mentioned to the author by A. Nerode].

THEOREM 4.2. Let $A, B \in \Lambda_{R}$. Then

(a) $D_{w}(A)$ is a prime regressive isol,

(b) $D_{w}(A)=D_{w}(B) \Rightarrow A=B$.

Proof. From the definition of the function $w(x)$ we note that each of the arithmetic statements

$$
\begin{aligned}
& w(a)=(u+2) \cdot v \Rightarrow v=1, \\
& w(a)=w(b) \Rightarrow a=b,
\end{aligned}
$$


is universally valid in E. Application of Lemmas A and B to (4.14) and (4.15), and substitution gives both

$$
D_{w}(A)=(U+2) \cdot V \Rightarrow V=1, \text { for } U, V \in \Lambda,
$$

and

$$
D_{w}(A)=D_{w}(B) \Rightarrow A=B .
$$

Part (b) follows directly from (4.17). To verify part (a), we first note that $D_{w}(A)$ is a regressive isol, because $A$ is a regressive isol. To show that $D_{w}(A)$ is also prime, assume that

$$
D_{w}(A)=T \cdot V
$$

for two isols $T$ and $V$. We wish to show that either $T=1$ or $V=1$. Because $D_{w}(A)$ will be nonzero it is readily seen that $T \neq 0$. If $T=1$, then we are done. Assume now that $T \neq 1$. Then $T \geqq 2$, and we let $U=T-2$. Then (4.18) can be expressed by,

$$
D_{w}(A)=(U+2) \cdot V .
$$

Finally combining (4.16) and (4.19) implies that $V=1$. It follows therefore that $D_{w}(A)$ will be a prime regressive isol. This verifies (a) and completes the proof.

REMARK. We note that by combining Theorem 4.2 with the fact that there are $c$ regressive isols, it follows that there will exist $c$ prime regressive isols belonging to $D_{w}\left(\Lambda_{R}\right)$. We wish also to note that the prime regressive isols obtained from Corollary 4.1 [these were infinite multiple-free regressive isols] will not belong to $D_{w}\left(\Lambda_{R}\right)$. This property will follow from the next result and the fact that infinite multiple-free isols are neither even nor odd.

THEOREM 4.3. Let $B \in \Lambda_{R}$. Then $D_{w}(B)=2$ if $B=0$, and $D_{w}(B)$ is an odd (regressive) isol if $B \geqq 1$.

Proof. If $B=0$, then the result is easy.

Assume now that $B \geqq 1$. We note that for each number $a \in E, w(a+1)$ will be an odd (prime) number. Let the function $u(a)$ be defined by,

$$
w(a+1)=2 u(a)+1, \quad \text { for } a \in E .
$$

It is readily seen that the function $u(a)$ is recursive and increasing. Therefore, its canonical extension $D_{u}$ will map $\Lambda_{R}$ into $\Lambda_{R}$. Combining Lemma A and (4.20), it follows that

$$
D_{w}(A+1)=2 D_{u}(A)+1, \text { for } A \in \Lambda_{R} .
$$

Because $B \in \Lambda_{R}$ and $B \geqq 1$, it follows that $B-1 \in \Lambda_{R}$ and hence also that $D_{u}(B-1)$ $\in \Lambda_{R}$. If we substitute $B-1$ for $A$ in (4.21), we obtain $D_{w}(B)=2 D_{u}(B-1)+1$, from which it follows that $D_{w}(B)$ will be an odd regressive isol. 
LEMMA C. Let $f: E \rightarrow E$ be an increasing recursive function, and $A$ and $B$ any regressive isols. Then

$$
A+B \in \Lambda_{R} \Rightarrow D_{f}(A)+D_{f}(B) \in \Lambda_{R} .
$$

Proof. Assume that $A+B$ is a regressive isol. Let $g: E \rightarrow E$ be an increasing recursive function chosen such that the recursive function $h(x)=f(x)+g(x)$, will be combinatorial. By Lemma $\mathrm{A}$ it follows that

$$
D_{h}(A)=D_{f}(A)+D_{g}(A), \quad D_{h}(B)=D_{f}(B)+D_{g}(B) .
$$

Because each of the recursive functions $f, g$ and $h$ is increasing, it follows that each of the isols appearing in (4.22) is regressive. In addition, $D_{h}(A+B)$ will also be a regressive isol, since $A+B \in \Lambda_{R}$. Consider the following implications,

$$
\begin{aligned}
A \leqq A+B & \Rightarrow D_{h}(A) \leqq D_{h}(A+B) \\
& \Rightarrow D_{f}(A)+D_{g}(A) \leqq D_{h}(A+B) \\
& \Rightarrow D_{f}(A) \leqq D_{h}(A+B) .
\end{aligned}
$$

The first implication follows from [20, Theorem 2] and the fact that since $h$ is a combinatorial function its first difference function will also be combinatorial and hence increasing. The second implication follows from (4.22), and the third one is clear. Clearly $A \leqq A+B$, and therefore,

$$
D_{f}(A) \leqq D_{h}(A+B) .
$$

In a similar manner it can be shown that

$$
D_{f}(B) \leqq D_{h}(A+B) .
$$

Since $D_{h}(A+B) \in \Lambda_{R}$, it follows from (2.1), (4.23) and (4.24) that

$$
D_{f}(A)+D_{f}(B) \in \Lambda_{R}
$$

and this is the desired result.

THEOREM 4.4. There exist distinct prime regressive isols $U_{1}, U_{2}, V_{1}$ and $V_{2}$ such that

(a) $U_{1}, U_{2}, \dot{V}_{1}, V_{2} \in D_{w}\left(\Lambda_{R}\right)$,

(b) $U_{1}+U_{2}=V_{1}+V_{2}$

(c) $U_{1} \cdot U_{2}=V_{1} \cdot V_{2}$,

(d) $U_{1}+U_{2}, U_{1} \cdot U_{2} \in \Lambda_{R}$.

Proof. By [3, Theorem 2.2], there will exist two (infinite) regressive isols that are not $\leqq *$ comparable and yet whose sum is a regressive isol. Let $A$ and $B$ denote two regressive isols of this kind. We now verify that the regressive isols

$$
\begin{array}{ll}
U_{1}=D_{w}(A), & V_{1}=D_{w}(\min (A, B)), \\
U_{2}=D_{w}(B), & V_{2}=D_{w}(\max (A, B)),
\end{array}
$$


will satisfy the conditions of the theorem. It follows from (6), (7), $(\alpha),(\beta)$ and $(\zeta)$, and the fact that $A$ and $B$ are not $\leqq *$ comparable that the four regressive isols $A, B, \min (A, B)$ and $\max (A, B)$ are distinct. By Theorem 4.2(b), this also means that the four regressive isols $U_{1}, U_{2}, V_{1}$ and $V_{2}$ are also distinct. In light of the definitions of $U_{1}, U_{2}, V_{1}$ and $V_{2}$, we see that part (a) follows directly. Part (b) follows from Theorems 2.3(a) and 3.1(c), and part (c) follows from parts (a) and (d) of Theorem 3.1. Lastly, combining Lemma C and (2.2) gives part (d).

REMARK. We wish to give an analogue for regressive isols of the following well-known theorem from number theory, called Bertrand's conjecture and proved by Chebyshev in 1850; see [14, p. 108]:

THEOREM A. For each number $a \in E$, there is a prime $p$ such that, $a<p \leqq 2 a$.

We will need two lemmas; the second of these is proved in [6].

LEMMA 4.1. Let $f, g: E \rightarrow E$ be increasing recursive functions. If

$$
(\forall t \in E)[f(t) \leqq g(t)]
$$

then

$$
\left(\forall T \in \Lambda_{R}\right)\left[D_{f}(T) \leqq D_{g}(T)\right] .
$$

Proof. Assume that $f(t) \leqq g(t)$, for each number $t$. Then

$$
\min (f(t), g(t))=f(t), \quad \text { for } t \in E .
$$

Therefore, by Lemma A, (7) and the fact that the canonical extensions of increasing recursive functions map $\Lambda_{R}$ into $\Lambda_{R}$, it follows that

and

$$
\min \left(D_{f}(T), D_{g}(T)\right)=D_{f}(T), \quad \text { for } T \in \Lambda_{R},
$$

$$
D_{f}(T) \leqq D_{g}(T), \quad \text { for } T \in \Lambda_{R}
$$

Lemma 4.2 [6, TheOREM 1(a)]. Let $f, g: E \rightarrow E$ be increasing recursive functions. Then

$$
f(E) \subseteq g(E) \Rightarrow D_{f}\left(\Lambda_{R}\right) \subseteq D_{g}\left(\Lambda_{R}\right)
$$

THEOREM 4.5. For each regressive isol $A$, there is a prime regressive isol $P$ such that $P \in D_{w}\left(\Lambda_{R}\right)$ and $A \leqq * P \leqq * 2 A$.

Proof. In view of Theorem A, it is easily seen that there will exist an increasing recursive function $p(a)$ such that, for each number $a \in E, a \leqq p(a) \leqq 2 a$, and $p(a)$ is a prime number.

Therefore, by Lemmas 4.1 and 4.2, it follows that for each regressive isol $A$,

$$
A \leqq{ }^{*} D_{p}(A) \leqq * 2 A \text { and } D_{p}(A) \in D_{w}\left(\Lambda_{R}\right) .
$$

Since $D_{w}\left(\Lambda_{R}\right)$ consists entirely of prime regressive isols, the desired result follows.

REMARK. Regarding Theorem 4.5, we do not know whether or not it is the case 
that for each regressive isol $A$, there is a prime regressive isol $P$ such that $P \in D_{w}\left(\Lambda_{R}\right)$ and $A \leqq P \leqq 2 A$.

\section{REFERENCES}

1. J. Barback, Recursive functions and regressive isols, Math. Scand. 15 (1964), $29-42$.

2. - A note on regressive isols, Notre Dame J. Formal Logic 7 (1966), 203-205.

3. - Two notes on regressive isols, Pacific J. Math. 16 (1966), 407-420.

4. —_ Double series of isols, Canad. J. Math. 19 (1967), 1-15.

5. - - Regressive upper bounds, Seminario Matematico 39 (1967), 248-272.

6. _- On recursive sets and regressive isols, Michigan Math. J. 15 (1968), 27-32.

7. J. C. E. Dekker, "Infinite series of isols" in Recursive function theory, Proc. Sympos.

Pure Math., Vol. 5, Amer. Math. Soc., Providence, R. I., 1962, pp. 77-96.

8. - The minimum of two regressive isols, Math. Z. 83 (1964), 345-366.

9. - "Regressive isols" in Sets, models and recursion theory, North-Holland, Amsterdam, 1967, pp. 272-296.

10. J. C. E. Dekker and J. Myhill, Recursive equivalence types, Univ. Calif. Purbl. Math. (N.S.) 3 (1960), 67-213.

11. E. Ellentuck, Review of "Extensions to isols", by A. Nerode (see [16]), Math. Reviews 24 (1962), \#A1215.

12. - Universal isols, Math. Z. 98 (1967), 1-8.

13. M. J. Hassett, Some theorems on regressive isols and isolic groups, Doctoral Thesis, Rutgers, The State University, New Brunswick, N. J., 1966.

14. W. J. LeVeque, Topics in number theory, Vol. 1, Addison-Wesley, Reading, Mass., 1956, pp. 1-198.

15. J. Myhill, Recursive equivalence types and combinatorial functions, Proc. 1960 Internat. Congress in Logic, Methodology and Philosophy of Science, Stanford, Stanford Univ. Press, Stanford, Calif., 1962, pp. 46-55.

16. A. Nerode, Extensions to isols, Ann. of Math. 73 (1961), 362-403.

17. —_ Extensions to isolic integers, Ann. of Math. 75 (1962), 419-448.

18. I. Niven and H. S. Zuckerman, An introduction to the theory of numbers, Wiley, New York, 1962, pp. 1-250.

19. F. J. Sansone, Combinatorial functions and regressive isols, Pacific J. Math. 13 (1963), 703-707.

20. —_, On order-preserving extensions to regressive isols, Michigan Math. J. 13 (1966), 353-355.

STANFORd UNIVERSITY,

Stanford, California

State University OF New York, Buffalo, New York 